\title{
Interventional Treatment of Severe Portal Vein Thrombosis after Living-Donor Liver Transplantation
}

\author{
Youichi Kawano ${ }^{1}$, Satoru Murata ${ }^{2}$, Nobuhiko Taniai ${ }^{1}$, Masato Yoshioka ${ }^{1}$, Atsushi Hirakata ${ }^{3}$, \\ Yoshiaki Mizuguchi ${ }^{1}$, Tetsuya Shimizu ${ }^{1}$, Tomohiro Kanda ${ }^{1}$, Junji Ueda ${ }^{1}$, Hideyuki Takada ${ }^{1}$, \\ Hiroshi Yoshida ${ }^{3}$, Koho Akimaru', Shiro Onozawa ${ }^{2}$, \\ Shinichirou Kumita ${ }^{2}$ and Eiji Uchida ${ }^{1}$
}

${ }^{1}$ Department of Gastrointestinal and Hepato-Biliary-Pancreatic Surgery, Nippon Medical School, Tokyo, Japan ${ }^{2}$ Department of Radiology, Nippon Medical School, Tokyo, Japan

${ }^{3}$ Department of Surgery, Nippon Medical School Tama Nagayama Hospital, Tokyo, Japan

Portal vein thrombosis (PVT) is a rare complication of liver transplantation which can lead to graft failure and patient death. Treatment can be difficult, especially in cases of PVT from the intrahepatic portal vein to the proximal jejunal veins. A 55-year-old woman had undergone living-donor liver transplantation with splenectomy for end-stage liver cirrhosis due to hepatitis $C$ with hepatocellular carcinoma. Ten months after transplantation, massive ascites and slight abdominal pain developed, and computed tomography revealed a PVT between the intrahepatic portal vein and the superior mesenteric vein. Repeated interventional radiology procedures were used in combination with thrombolysis, thrombectomy, and metallic stent replacement to obtain favorable portal flow to the graft. Five years after being treated, the patient is well, with favorable portal flow having been confirmed. In conclusion, repeated and assiduous interventional radiological treatment combined with thrombolytic therapy, thrombectomy, and metallic stent replacement could be important for severe PVT.

(J Nippon Med Sch 2016; 83: 206-210)

Key words: living-donor liver transplantation, portal vein thrombosis, complication, interventional treatment

\section{Introduction}

Portal vein thrombosis (PVT) is a rare complication of liver transplantation (LT) which can lead to graft failure and patient death. Because PVT can cause symptoms of portal hypertension, such as a rupture of esophageal varices or ascites, both its diagnosis and treatment are important. However, the treatment of PVT is difficult and complicated, especially when the PVT extends from the intrahepatic to the proximal superior mesenteric vein (SMV). Significant progress has been made in interventional radiology procedures regarding complications following LT, and these procedures should be used to treat PVT even in cases of severe PVT after LT. Therefore, we report a 55-year-old woman with severe portal vein thrombosis after living-donor liver transplantation who could treat by repeated, assiduous and precise interventional radiological treatments combined with thrombolytic therapy.

\section{Case Report}

A 55-year-old woman underwent living-donor LT with splenectomy at our hospital in June 2008 because of endstage liver cirrhosis due to hepatitis $C$ with hepatocellular carcinoma. The patient did not have PVT at the time of the operation, and a left lobe from the patient's son was used for the graft. Portal vein reconstruction was performed via end-to-end continuous anastomosis with a 6-0 monofilament suture and resulted in a graft portal vein orifice $15 \mathrm{~mm}$ in diameter. For immunosuppression tacrolimus and methylprednisolone were administered.

Correspondence to Youichi Kawano, MD, Department of Gastrointestinal and Hepato-Biliary-Pancreatic Surgery, Nippon Medical School, 1-1-5 Sendagi, Bunkyo-ku, Tokyo 113-8603, Japan

E-mail: y-kawano@nms.ac.jp

Journal Website (http://www.nms.ac.jp/jnms/) 

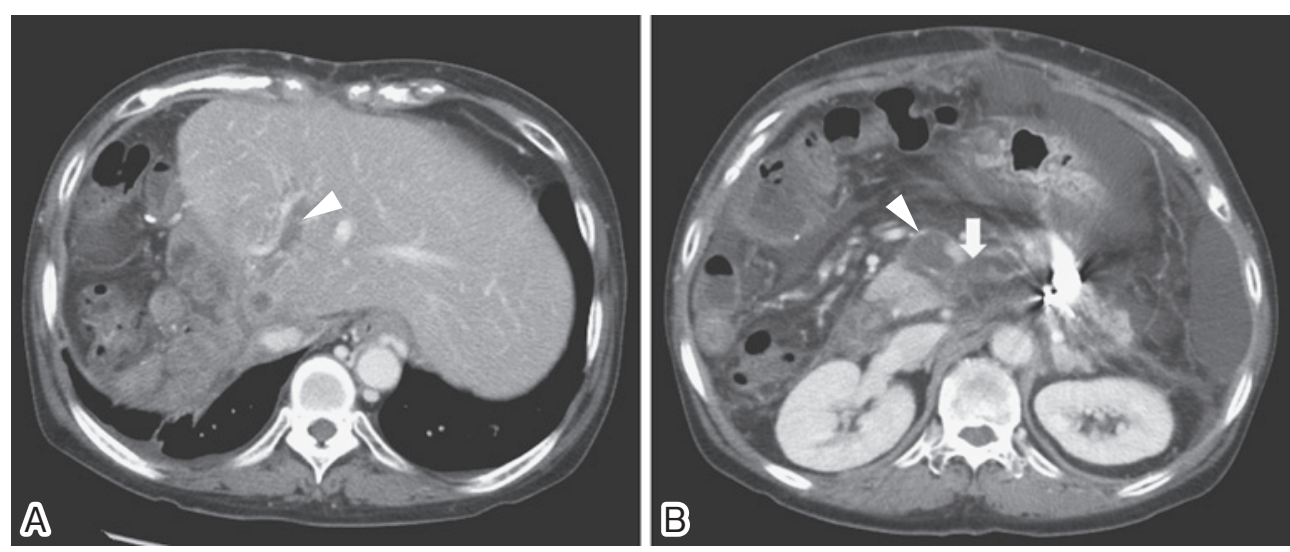

Fig. 1 Emergency computed tomography on admission revealed a portal vein thrombus between the intrahepatic portal vein (A, arrow) and the superior mesenteric vein (B, arrowhead), which extended to the jejunal veins (B, arrow).

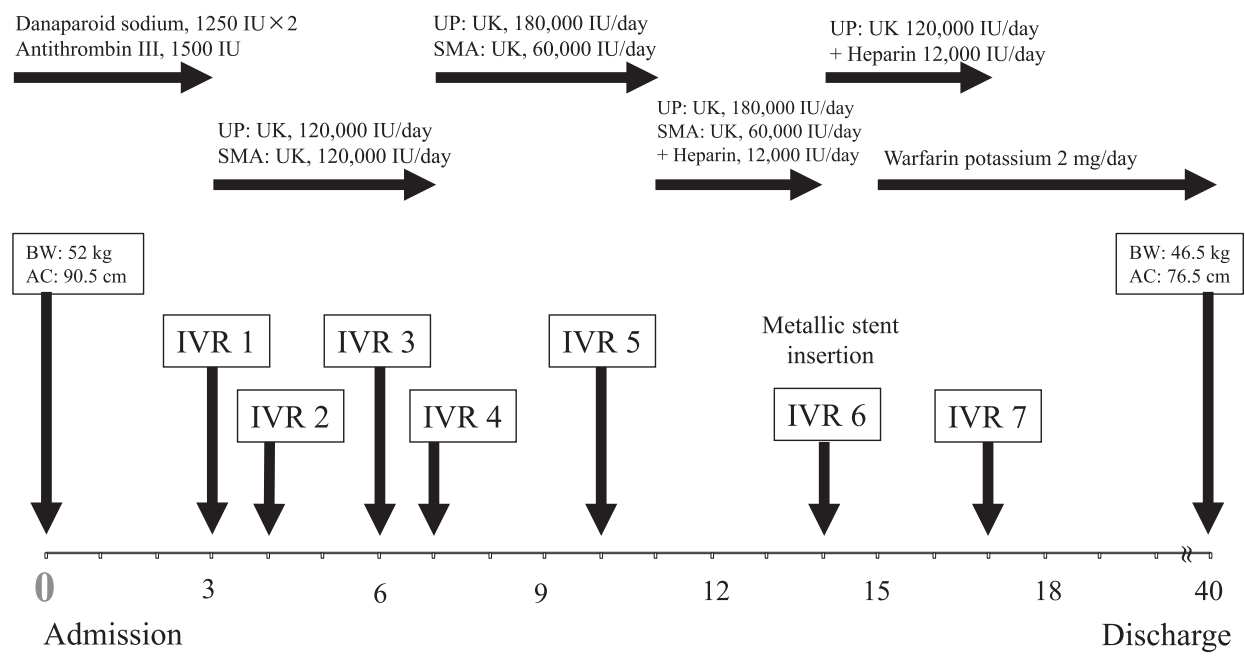

Hospital Day

Fig. 2 The diagram shows the sequence of combined treatments for portal vein thrombosis in our hospital. Abbreviations: UP, umbilical portion; UK, urokinase; SMA, superior mesenteric artery; IVR, interventional radiology; BW, body weight; AC, abdominal circumference

On day 10 after LT, bleeding from the abdominal drains suddenly occurred and caused the patient to go into shock. Emergency arterial embolization with interventional procedures was performed to rupture the right subphrenic artery and achieved complete hemostasis. The subsequent postoperative course was uneventful, and the patient was discharged on day 88 after LT.

Six months after LT, combination therapy for hepatitis $C$ was started with peginterferon $\alpha-2 b$ and ribavirin. Computed tomography (CT) revealed moderate portal vein stenosis (PVS) without a thrombus, but suitable for portal perfusion. Because massive ascites with liver dysfunction and slight abdominal pain developed 3 months after treatment, the patient was re-admitted to the hospi- tal for assessment and treatment 10 months after LT. A CT examination revealed massive ascites and a PVT that was between the intrahepatic portal vein and the SMV and extended to the jejunal veins (Fig. 1A, B). However, major collateral veins around the hepatic hilum and the SMV were unable to be used for portal vein reconstruction. We chose not to performed operative treatment for the PVT because the thrombus extended to the intrahepatic portal vein; instead we decided to perform interventional radiology procedures (Fig. 2).

In the first interventional radiology procedure, a radiologist punctured the umbilical portion of the liver, which had hyperechoic material in the lumen, under ultrasonographic guidance and confirmed with Doppler ul- 

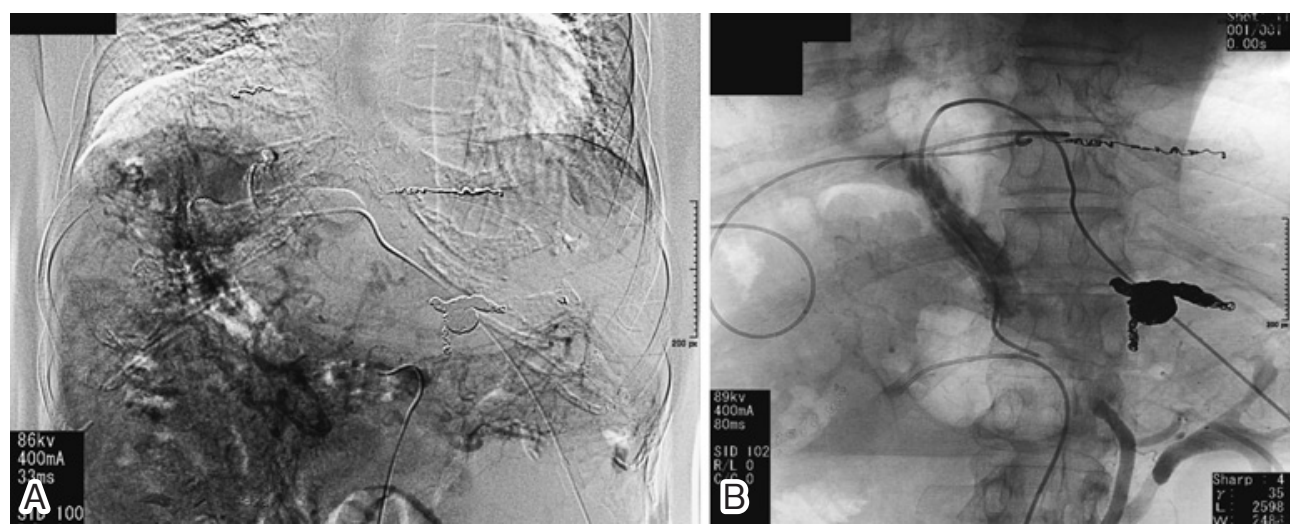

Fig. 3 Arterial portography revealed tiny collateral vessels and total occlusion of the portal venous system (A). Direct portography revealed total occlusion of the portal venous system, including the graft, by a thrombus (B).

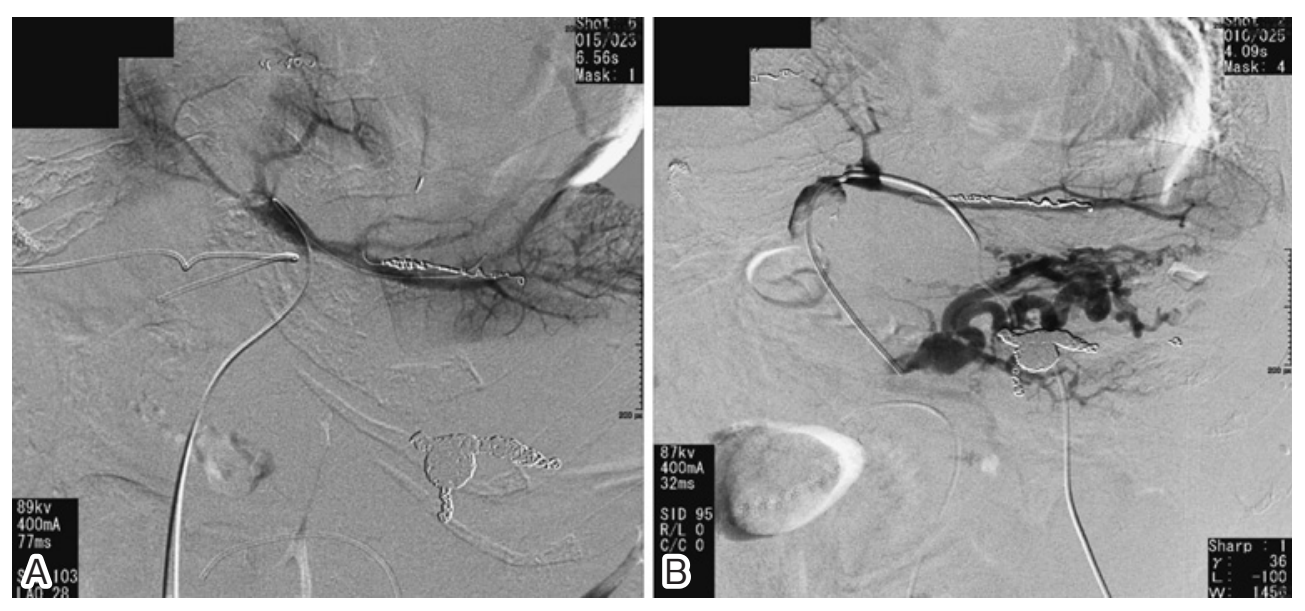

Fig. 4 The intrahepatic portal vein was recanalized in the fourth session of interventional radiological treatment (A). Furthermore, penetration of a catheter through the thrombus from the umbilical portion to the distal jejunal veins could be done in the same procedure (B).

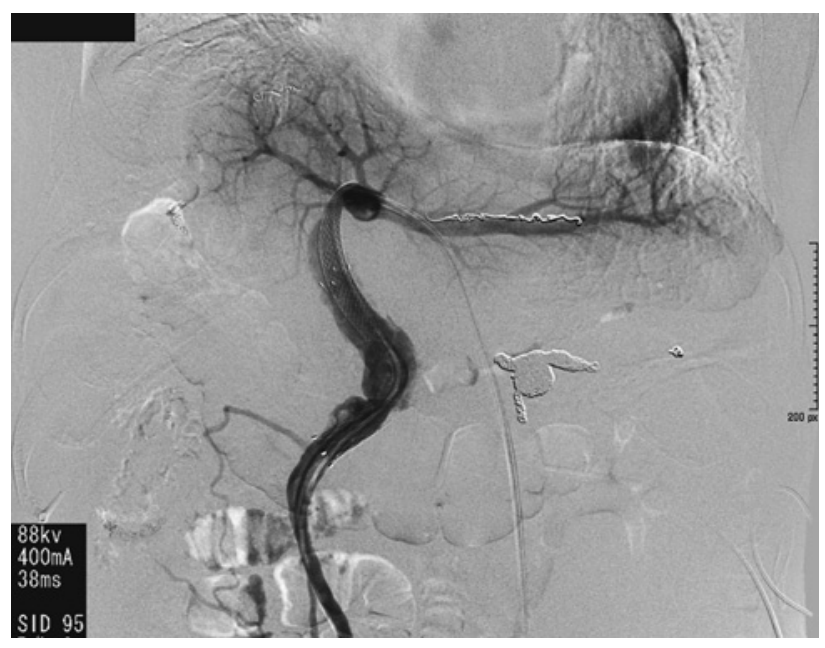

Fig. 5 Confirmation angiography after the seventh interventional radiological procedure revealed improvement of the stenosis. trasonography the absence of portal flow. Arterial portography revealed tiny collateral vessels and total occlusion of the portal venous system (Fig. 3A). Direct portography revealed total occlusion of the portal venous system, including the graft, by a thrombus (Fig. 3B). Catheters were placed into the umbilical portion and the superior mesenteric artery, and urokinase was continuously administered at the rate of $120,000 \mathrm{U} /$ day.

In the second to fifth interventional radiological procedures, repeated thrombectomy and thrombolysis were performed. We were able to penetrate a catheter through the thrombus from the umbilical portion to the patient's jejunal vein and recanalize the intrahepatic portal vein in the fourth treatment session (Fig. 4A, B).

In the sixth interventional radiological procedure, a 10cm-long segment of a 10-mm-diameter E-Luminexx ${ }^{\mathrm{TM}}$ vascular stent (Bard Peripheral Vascular, Tempe, AZ, 
USA) was placed in the portal vein extending to the SMV, and a 6-cm-long segment of a 10-mm-diameter Wallstent $^{\mathrm{TM}}$ stent (Boston Scientific Corp., Marlborough, MA, USA) was also placed from the intrahepatic portal vein to the portal vein by means of the partial stent-instent technique.

In the seventh interventional radiological procedure, confirmation angiography revealed improvement of the stenosis (Fig. 5).

Several days after the seventh procedure CT revealed a marked decrease in ascites, and favorable perfusion of the contrast medium from the SMV to peripheral intrahepatic portal veins without thrombus in the stents were observed. After the patient was discharged, warfarin potassium was administered, and a target international normalized ratio of approximately 2 was set for the rest of the patient's life. Five years after treatment, the patient is well and without ascites or liver dysfunction, and favorable portal flow has been confirmed with ultrasonography.

\section{Discussion}

Reported risk factors for PVT include the causes of many cases of PVT following LT, smaller graft size, pre-existing PVT, small diameter of the portal vein, pre-LT surgical shunt operation, splenectomy, PVS, and use of either jump or interposition grafts for portal vein reconstruction $^{1,2}$. In the present case, the patient had several of these risk factors for PVT, such as a previous LT, PVS, and splenectomy.

A case of PVT after LT can be categorized as "early" (detected within 3 months after LT) or "late" (detected more than 3 months after LT) ${ }^{3}$. Furthermore, PVT is clinically classified as "acute" if symptoms developed within 60 days before hospital assessment or as "chronic" if symptoms developed after that ${ }^{1,4}$. For late PVT, the management is less well defined because the clinical implications vary and range from asymptomatic, due to sufficient portal flow being derived from collateral veins around the hepatic hilum, to symptomatic, such as massive ascites and gastrointestinal bleeding due to portal hypertension. Acute PVT can easily be differentiated from chronic PVT by portoportal collateral vessels being absent or insignificant on imaging studies and by a lack of evidence for portal hypertension, including splenomegaly and esophageal varices. In the present case, the PVT could be clinically classified as late and acute. The patient had massive ascites, slight liver dysfunction, and abdominal pain that were suspected to have been caused by intestinal congestion. Furthermore, CT revealed only fine collateral veins around the PVT. Because the development of collateral veins in short time to circumvent the PVT as a compensatory mechanism called "venous rescue" for the portal vein obstruction completes within 3 to 5 weeks ${ }^{5}$, we diagnosed acute PVT in the patient. Cases of PVT have been classified into 4 categories depending on the extent of thrombosis ${ }^{6}$. Cases of type IV, as the present PVT could be classified, may have the worst prognosis.

Treatments for PVT are generally chosen from systemic or local thrombolysis, surgical treatment such as thrombectomy, portosystemic shunting, or percutaneous intervention. In a case of the entire PVT extending to the intrahepatic portal vein and the branch of the SMV having symptomatic conditions, the treatment would be difficult and complicated. In the present case, although urgent treatment was required, deciding which treatment to perform was difficult. Shunt surgeries creating a portal or portosystemic shunt were rejected because there was no adequate place to create the shunt in the graft. Although only a few cases with interventional radiological treatment for PVT after LT have been reported ${ }^{7-9}$, this technique is appropriate for many complications following LT because of its minimal invasiveness, low rate of complication, and high success rate ${ }^{2}$. Especially in PVT after LT, combination therapy, including via systemic venous circulation, the superior mesenteric artery, or the transhepatic portal vein; endovascular thrombectomy and stent replacement seem to be effective $\mathrm{e}^{10,11}$. We also decided to perform combination therapy for the severe PVT with thrombolysis and transhepatic approach thrombectomy, passing through the thrombus with a guide-wire from the intrahepatic portal vein to the distal jejunal vein, and balloon dilatation and stent replacement for the PVS.

To prevent PVT in the present patient, interventional radiological balloon dilatation or anticoagulation therapy should have be performed when the PVS had been determined with CT. Periodic imaging examination with Doppler ultrasonography or enhanced CT or both and preemptive balloon dilatation could be extremely important to avoid PVT following late onset PVS after $\mathrm{LT}^{2,3,12}$.

\section{Conclusion}

Repeated, assiduous and precise interventional radiological treatments combined with thrombolytic therapy, thrombectomy, and metallic stent replacement could be important for treating severe PVT extending from the intrahepatic to the proximal SMV without a steady collat- 
eral vein after LT. Further studies are needed to determine the optimal treatment of severe PVT.

Conflict of Interest: The authors declare no conlict of interst.

\section{References}

1. Chawla Y, Duseja A, Dhiman RK: Review article: the modern management of portal vein thrombosis. Aliment Pharmacol Ther 2009; 30: 881-894.

2. Kawano Y, Mizuta K, Sugawara Y, Egami S, Hisikawa S, Sanada Y, Fujiwara T, Sakuma Y, Hyodo M, Yoshida Y, Yasuda Y, Sugimoto E, Kawarasaki H: Diagnosis and treatment of pediatric patients with late-onset portal vein stenosis after living donor liver transplantation. Transpl Int 2009; 22: 1151-1158.

3. Kyoden Y, Tamura S, Sugawara Y, Matsui Y, Togashi J, Kaneko J, Kokudo N, Makuuchi M: Portal vein complications after adult-to-adult living donor liver transplantation. Transpl Int 2008; 21: 1136-1144.

4. DeLeve LD, Valla DC, Garcia-Tsao G: Vascular disorders of the liver. Hepatology 2009; 49: 1729-1764.

5. Ponziani FR, Zocco MA, Campanale C, Rinninella E, Tortora A, Di Maurizio L, Bombardieri G, De Cristofaro R, De Gaetano AM, Landolfi R, Gasbarrini A: Portal vein thrombosis: insight into physiopathology, diagnosis, and treatment. World J Gastroenterol 2010; 16: 143-155.

6. Jamieson NV: Changing perspectives in portal vein thrombosis and liver transplantation. Transplantation 2000; 69: 1772-1774.
7. Durham JD, LaBerge JM, Altman S, Kam I, Everson GT, Gordon RL, Kumpe DA: Portal vein thrombolysis and closure of competitive shunts following liver transplantation. J Vasc Interv Radiol 1994; 5: 611-615.

8. Carnevale FC, Borges MV, Moreira AM, Cerri GG, Maksoud JG: Endovascular treatment of acute portal vein thrombosis after liver transplantation in a child. Cardiovasc Intervent Radiol 2006; 29: 457-461.

9. Nosaka S, Isobe Y, Kasahara M, Miyazaki O, Sakamoto S, Uchida H, Shigeta T, Masaki H: Recanalization of posttransplant late-onset long segmental portal vein thrombosis with bidirectional transhepatic and transmesenteric approach. Pediatr Transplant 2013; 17: E71-E75.

10. Cherukuri R, Haskal ZJ, Naji A, Shaked A: Percutaneous thrombolysis and stent placement for the treatment of portal vein thrombosis after liver transplantation: longterm follow-up. Transplantation 1998; 65: 1124-1126.

11. Baccarani U, Gasparini D, Risaliti A, Vianello V, Adani GL, Sainz M, Sponza M, Bresadola F: Percutaneous mechanical fragmentation and stent placement for the treatment of early posttransplantation portal vein thrombosis. Transplantation 2001; 72: 1572-1582.

12. Ueda M, Oike F, Kasahara M, Ogura Y, Ogawa K, Haga H, Takada Y, Egawa H, Tanaka K, Uemoto S: Portal vein complications in pediatric living donor liver transplantation using left-side grafts. Am J Transplant 2008; 8: 20972105.

(Received, March 23, 2016)

(Accepted, September 20, 2016) 